\title{
Safety and efficacy of the endothelin receptor antagonist macitentan in pediatric pulmonary hypertension
}

\author{
Sabrina Schweintzger ${ }^{1,2}$, Martin Koestenberger ${ }^{1,2}$, Axel Schlagenhauf ${ }^{3}$, Gernot Grangl ${ }^{1}$, Ante Burmas ${ }^{1}$, \\ Stefan Kurath-Koller ${ }^{1}$, Mirjam Pocivalnik ${ }^{4}$, Hannes Sallmon ${ }^{2,5}$, Daniela Baumgartner ${ }^{1}$, Georg \\ Hansmann $^{2,6}$, Andreas Gamillscheg ${ }^{1}$
}

${ }^{1}$ Division of Pediatric Cardiology, Department of Pediatrics, Medical University of Graz, Austria; ${ }^{2}$ European Pediatric Pulmonary Vascular Disease Network, Berlin, Germany; ${ }^{3}$ Division of General Pediatrics and Adolescent Medicine, ${ }^{4}$ Pediatric Intensive Care Unit, Department of Pediatrics, Medical University of Graz, Graz, Austria; ${ }^{5}$ Department of Pediatric Cardiology, Charité University Medical Center, Berlin, Germany; ${ }^{6}$ Department of Pediatric Cardiology and Critical Care, Hannover Medical School, Hannover, Germany

Contributions: (I) Conception and design: S Schweintzger, A Gamillscheg, M Koestenberger; (II) Administrative support: S Kurath-Koller, M Pocivalnik, H Sallmon, G Hansmann; (III) Provision of study materials or patients: G Grangl, A Burmas; (IV) Collection and assembly of data: D Baumgartner; (V) Data analysis and interpretation: A Schlagenhauf; (VI) Manuscript writing: All authors; (VII) Final approval of manuscript: All authors.

Correspondence to: Sabrina Schweintzger, MD; Andreas Gamillscheg, MD. Division of Pediatric Cardiology, Department of Pediatrics, Medical University Graz, Auenbruggerplatz 34/2, A-8036 Graz, Austria. Email: sabrina.schweintzger@medunigraz.at; andreas.gamillscheg@medunigraz.at.

Background: Macitentan, a dual endothelin receptor antagonist (ERA), was approved in 2014 for the treatment of adults with idiopathic pulmonary arterial hypertension (PAH). Once-per-day dosing and low potential hepatic toxicity make macitentan an appealing therapeutic option for children with PAH, but reports on its use in pediatric patients are still lacking.

Methods: Prospective observational study of 18 children $[10$ male; median age: 8.5 , minimum (min.): 0.6, maximum (max.): 16.8 years] with pulmonary hypertension $(\mathrm{PH})$. Four of these 18 patients were treatmentnaïve and started on a de novo macitentan therapy. The remaining 14/18 children were already on a $\mathrm{PH}-$ targeted pharmacotherapy (sildenafil or bosentan as monotherapy or in combination). Nine children who were on bosentan were switched to macitentan. We analyzed the 6-minute walking distance (6MWD), NYHA functional class (FC)/modified ROSS score, invasive hemodynamics, echocardiographic variables and the biomarker N-terminal pro-brain natriuretic peptide (NT-proBNP).

Results: The median follow up was 6 months (min.: 0.5, max.: 30). Macitentan treatment was associated with improvement of invasive hemodynamics, e.g., the ratio of mean pulmonary arterial pressure/mean systemic arterial pressure decreased from a median of $62 \%$ (min.: $30 \%$, max.: $87 \%$ ) to $49 \%$ ( $\min .: 30 \%$, max.: $69 \%$ ), $\mathrm{P}<0.05$; pulmonary vascular resistance index (PVRi) decreased from a median of 7.6 (min.: 3.3, max.: 11.5) to 4.8 Wood units $\times \mathrm{m}^{2}$ body surface area (min.: 2.5, max.: 10), $\mathrm{P}<0.05$. The tricuspid annular plane systolic excursion (TAPSE) increased from a median of 1.4 (min.: 0.8, max.: 2.8 ) to 1.9 (min.: 0.8, max.: 2.7) cm, $(\mathrm{P}<0.05)$. NTproBNP values decreased from a median of 272 (min.: 27, max.: 2,010) to 229 (min.: 23, max.: 814) pg/mL under macitentan therapy $(\mathrm{P}<0.05)$. The 6MWD and NYHA FC/modified ROSS score did not change significantly.

Conclusions: This is the first prospective study of macitentan pharmacotherapy in infants and children with $\mathrm{PH}<12$ years of age. Except in one patient, macitentan treatment was well tolerated and was associated with improvements in invasive hemodynamics, longitudinal systolic RV function (TAPSE) and serum NTproBNP values.

Keywords: Bosentan; child; endothelin receptor antagonist (ERA); macitentan; pulmonary hypertension (PH)

Submitted Feb 10, 2020. Accepted for publication Mar 31, 2020.

doi: $10.21037 / \mathrm{cdt} .2020 .04 .01$

View this article at: http://dx.doi.org/10.21037/cdt.2020.04.01 


\section{Introduction}

Pulmonary hypertension $(\mathrm{PH})$ is a progressive, life-limiting condition impacting the patient's exercise capacity and quality of life, especially in the advanced disease stages (1). At the most recent World Symposium on Pulmonary Hypertension (WSPH 2018, Nice), PH in adults and children was redefined as an increase in mean pulmonary artery pressure $(\mathrm{mPAP})>20 \mathrm{mmHg}$ at rest, as assessed by cardiac catheterization $(2,3)$. Pulmonary arterial hypertension $(\mathrm{PAH})$ is characterized hemodynamically by pre-capillary $\mathrm{PH}$, defined by a pulmonary artery wedge pressure $\leq 15 \mathrm{mmHg}$ and a pulmonary vascular resistance index (PVRi) $>3$ Wood units in the absence of other causes of precapillary $\mathrm{PH}$ such as $\mathrm{PH}$ due to lung diseases, chronic thromboembolic $\mathrm{PH}$ or other rare diseases (4). Pediatric PAH is a rare condition that affects 2-16 per million children (5-7). Prior to sufficient treatment options, the median survival after diagnosis of patients with idiopathic PH (IPAH) was 10 months in children and 2.8 years in adults (8). The development of second-generation vasodilatory medications [prostanoids, 5 -phosphodiesterase inhibitors (PDE-5i), endothelin receptor antagonists (ERA)] coincided with marked clinical improvement or stabilization in patients with $\mathrm{PH}$. The combined adult and pediatric U.S. REVEAL registry (9) demonstrated 1-, 3- and 5-year estimated survival rates of $96 \% \pm 4 \%, 84 \% \pm 5 \%$ and $74 \% \pm 6 \%$, respectively for children with PAH [the IPAH and PAH associated with congenital heart disease (CHD) subgroups combined accounted for 199 of the total 216 patients; 92\%; IPAH, $\mathrm{n}=122$; PAH-CHD, $\mathrm{n}=77]$. Another report (10) found the 3 -year survival in children with PAH (29 children with IPAH and 23 children with shunt associated PAH) to be in a similar range $(83 \%)$. Although therapeutic options have increased over the past several decades, they remain limited in children. Only sildenafil (PDE-5i) and bosentan (ERA) have been approved for pediatric use in N. America and Europe to date. In 2001, with the approval of orally administered bosentan, treatment options improved greatly in adult PAH (11-14). To increase the safety and efficacy of bosentan, macitentan an oral, dual (non-selective) ERA was developed $(15,16)$. The pharmacokinetics of macitentan are characterized by slow absorption and a plasma a halflife of 17.5 hours at the maximum tolerated dose of 300 $\mathrm{mg}$, i.e., compatible with a once-a-day dosing regimen. The pharmacologically active metabolite of macitentan, ACT-132577, is formed by oxidative depropylation of macitentan, a reaction catalyzed by the cytochrome P450 isoenzyme, CYP3A4 (17). ACT-132577 was approximately five times less potent than macitentan (16). Due to its long half-life of about 48 hours, this metabolite is prone to accumulate upon repeated dosing and, therefore, significantly contributes to the overall effect (18). In the large event-driven SERAPHIN trial ( $\mathrm{n}=742$ total), oral macitentan $(10 \mathrm{mg})$ was well tolerated and reduced morbidity and mortality (composite end point of death, atrial septostomy, lung transplantation, initiation of treatment with intravenous or subcutaneous prostanoids, or worsening of PAH (HR 0.55 vs. placebo; $97.5 \%$ CI, 0.39 to $0.76 ; \mathrm{P}<0.001)$ among 242 adult $\mathrm{PAH}$ patients $(\mathrm{n}=134$ IPAH, 36.4\% treatment naïve) (19). Comparative studies for add-on bosentan $v s$. add-on macitentan do not exist, however, many $\mathrm{PH}$ centers probably consider these two unselective ERAs as equally effective until proven otherwise. Compared with bosentan, macitentan has several benefits, such as once-a-day dosing as well as lower risks of both drug-drug interactions and hepatotoxicity $(12,15,19-21)$. Using macitentan in combination with sildenafil may be superior since bosentan significantly decreases the plasma concentration of sildenafil when co-administered to patients with PH $(22,23)$. In addition, freedom from mandatory monthly liver function tests, as requested for bosentan, would be ideal and beneficial, especially in children (24). Experience with macitentan in the treatment of $\mathrm{PH}$ in children is very sparse $(19,25,26)$. Published information on the safety and efficacy of macitentan in the treatment of $\mathrm{PH}$ in infants and children $<12$ years is lacking completely. The aim of this prospective observational analysis is to present our first experience on the safety and efficacy of macitentan in the treatment of children with $\mathrm{PH}$ of different etiologies, from infancy to adolescence.

\section{Methods}

This is a single-center, prospective observational study on the safety and efficacy of oral macitentan in children with $\mathrm{PH}$ at the Division of Pediatric Cardiology, Department of Pediatrics at the Medical University of Graz, Austria [2015-2018]. Written informed consent was obtained from the legal caregivers of the patients.

\section{Study subjects}

We enrolled 18 children, $<18$ years (y) of age with 
confirmed $\mathrm{PH}$ prior to macitentan initiation, based on the invasive hemodynamic definition of the World Symposium on PH 2018 (2,3). Before starting macitentan therapy, we analyzed 6-minute walking distance (6MWD), New York Heart Association (NYHA) Functional Classification (FC)/modified ROSS score, invasive hemodynamics, echocardiographic variables and laboratory values.

After macitentan initiation [at a median follow-up of 6 months (mo), minimum (min.): 0.5, maximum (max.): $30 \mathrm{mo}$ ], we analyzed six-minute walking distance (6MWD), NYHA FC/modified ROSS score, invasive hemodynamics (possible in only 8 children because parents denied re-catheterization), echocardiographic variables and laboratory values.

\section{Study design and macitentan dosing}

Macitentan was administered orally once daily. The starting dose was adult dosing with 10 milligram ( $\mathrm{mg}$ ) in 15 children with a median age of $10.3 \mathrm{y}$ (min. $2.8 \mathrm{y}$; max. $16.8 \mathrm{y}$ ) and a median bodyweight (BW) of 24.8 kilogram $(\mathrm{kg})$ [ $\mathrm{min}$. $11.3 \mathrm{~kg}$, max. $106.5 \mathrm{~kg}$ ], equaling a median dose $/ \mathrm{kg} \mathrm{BW}$ of $0.4 \mathrm{mg} / \mathrm{kg}$ (min. dose $/ \mathrm{kg}$ BW: 0.09; max. dose $/ \mathrm{kg} \mathrm{BW:}$ $0.9)$. The starting dose was $5 \mathrm{mg}$ in 3 patients (median age: 0.9 y; min.: 0.6 y; max.: 1.3 y, median BW: 8.2 ; min.: $5 \mathrm{~kg}$; max.: $9.2 \mathrm{~kg}$ ), with a median dose $/ \mathrm{kg} B W$ of $0.6 \mathrm{mg} / \mathrm{kg}$ (min.: 0.5 ; max.: 1). The macitentan dose was subsequently escalated to $10 \mathrm{mg}$ in those who tolerated the drug well and reached $>10 \mathrm{~kg}$ body weight $(\mathrm{n}=2)$. All patients $(\mathrm{n}=18)$ underwent outpatient examinations at regular time intervals of 3 months, starting 2 weeks after initiation of macitentan therapy. Safety and tolerability were evaluated, based on clinical findings, recorded adverse events and laboratory parameters. Echocardiography and NYHA FC/modified ROSS score were performed in all patients. Hemodynamic data and a $6 \mathrm{MWD}$ were obtainable in 8 patients.

\section{Clinical assessment}

All patients underwent assessment of NYHA FC/modified ROSS score $(27,28)$ by two pediatric cardiologists.

\section{MWD}

The $6 \mathrm{MWD}$ was pursued according to the American Thoracic Society guidelines (29), before (median age: $10 \mathrm{y}$, min.: $5.5 \mathrm{y}$, max.: $15.4 \mathrm{y}$ ) and after initiation of macitentan therapy (median age: $11.6 \mathrm{y}$; min.: $6.1 \mathrm{y}$, max.:
$17 \mathrm{y})$ in 8 patients old enough to perform the test reliably.

\section{Laboratory tests}

Laboratory work-up included liver function tests (aspartate aminotransferase and alanine aminotransferase, gammaglutamyltransferase, bilirubin), hemoglobin, and N-terminal pro-brain natriuretic peptide (NT-proBNP). Increasing serum liver enzyme concentrations were defined as a twofold increase of age-dependent normal values.

\section{Transthoracic echocardiography}

All patients underwent transthoracic echocardiography before and after the initiation of macitentan therapy. Echocardiographic parameters included tricuspid annular plane systolic excursion (TAPSE), pulmonary acceleration time (PAAT) and estimated right ventricular systolic pressure gradient (RVSP). Echocardiograms were performed using a commercially available echocardiographic system (Sonos iE33, Philips, Andover, Mass., USA) using transducers of 5-1, 8-3 and 12-4 MHz depending on the patient's age, size and weight. Images were recorded digitally and analyzed using off-line software (Xcelera Echo; Philips Medical Systems, The Netherlands). All measures were averaged over 3 cycles and analyzed by 2 blinded certified readers (30). The TAPSE reflects the longitudinal excursion of the tricuspid annulus toward the apex and was measured with $\mathrm{M}$-mode in the apical 4-chamber view as described before (31). The PAAT was measured as the interval between the onset of ejection and the peak flow velocity, defined as the time from the onset to maximal velocity (32) and is an inverse surrogate of pulmonary artery pressure and pulmonary vascular resistance index (PVRi). The RVSP was assessed by tricuspid regurgitation velocity (TRV) calculated by applying the modified Bernoulli equation $\left[\Delta \mathrm{P}=4\left(\mathrm{~V}^{2}-\mathrm{V} 1^{2}\right)\right]$. All patients had measurable mild-to-moderate tricuspid regurgitation (TR) so that TR jets could be well interrogated with continuous wave Doppler. A TRV $>2.8 \mathrm{~m} / \mathrm{s}$ is considered a reasonable cutoff to define elevated pulmonary pressure in the absence of pulmonary stenosis $(33,34)$. Of note the right atrial v-wave (ca. $5-10 \mathrm{mmHg}$ ), which we could not measure invasively at the time of the echocardiogram, must be added to the estimated pressure gradient between RA and RV via the $\mathrm{CW}$-Doppler interrogation. All values are either TRV in $\mathrm{m} / \mathrm{s}$ or RVSP in $\mathrm{mmHg}$ (not adding a virtual RA v wave). 
Table 1 Demographic data of our children with pulmonary hypertension $(\mathrm{PH})$

\begin{tabular}{|c|c|}
\hline Demographic data & $\begin{array}{l}\text { N or median } \\
(\min . / \max .)\end{array}$ \\
\hline \multicolumn{2}{|l|}{ All patients } \\
\hline Number (n) & 18 \\
\hline Female, n (\%) & $8(44.4)$ \\
\hline Age at baseline (years) & $8.5(0.6-16.8)$ \\
\hline Body weight (kg) & $21.3(5.0-106.5)$ \\
\hline Body length (cm) & $124.3[55-192]$ \\
\hline \multicolumn{2}{|l|}{$\mathrm{PH}$ categories } \\
\hline $\mathrm{PAH}-\mathrm{CHD}$ (group 1.4.4) & 3 \\
\hline Complex PH-CHD (group 5.4) & 8 \\
\hline PH BPD (group $3.5 \mathrm{PH}$ ) & 5 \\
\hline PAH-portal hypertension (group 1.4.3 PH) & 1 \\
\hline $\mathrm{IPAH}$ (group 1.1) & 1 \\
\hline PH medication before macitentan (pre-MAC) & 18 \\
\hline None (treatment naïve) & 4 \\
\hline Bosentan & 1 \\
\hline Bosentan + sildenafil & 8 \\
\hline Sildenafil & 5 \\
\hline Macitentan treatment & 18 \\
\hline Start macitentan therapy (treatment naïve) & 4 \\
\hline $\begin{array}{l}\text { Switch monotherapy bosentan to macitentan } \\
\text { monotherapy }\end{array}$ & 1 \\
\hline $\begin{array}{l}\text { Switch combination bosentan and sildenafil to } \\
\text { macitentan and sildenafil }\end{array}$ & 8 \\
\hline Macitentan add-on to sildenafil & 5 \\
\hline PH medication at end of study (post-MAC) & 18 \\
\hline Macitentan & 2 \\
\hline Macitentan and sildenafil & 16 \\
\hline
\end{tabular}

Age of our patients at baseline is the age at inclusion in the study. $\mathrm{PAH}$, pulmonary arterial hypertension; $\mathrm{PAH}-\mathrm{CHD}, \mathrm{PAH}$ associated with congenital heart disease; $\mathrm{PH}$, pulmonary hypertension; $\mathrm{PH}-\mathrm{BPD}, \mathrm{PH}$ due to bronchopulmonary dysplasia; IPAH, idiopathic PAH; MAC, macitentan; min., minimum; max., maximum.

\section{Invasive hemodynamic assessment}

Right and left heart cardiac catheterization was performed under general anesthesia. All patients underwent cardiac catheterization before the start of macitentan, to confirm PH. In eight patients with biventricular circulation, cardiac catheterization before and after macitentan initiation was performed (the remaining parents denied re-catheterization). The invasive measurements included mean pulmonary artery pressure (mPAP), ratio of mean pulmonary artery pressure/ mean systemic artery pressure ( $\mathrm{mPAP} / \mathrm{mSAP}$ ratio), with calculation of PVRi and cardiac index (CI).

\section{Ethics}

This study complies with the institutional guidelines related to patient confidentiality and research ethics including the institutional review board approval of the Ethics Board of Graz Medical University (Ethics Committee Number 30-477 ex 17/18).

\section{Statistics}

Data are reported via descriptive statistics, i.e., absolute and relative frequencies for categorical value and median, minimum, maximum and sample size. Due to the small sample size, all data are presented as median, min. and max. The significance of changes between start and follow-up visits was tested by the non-parametric Wilcoxon test and Fisher's exact test for categorical parameters. $\mathrm{P}<0.05$ was considered significant. All data were analyzed as recorded without imputation of missing values. Stata 14.2 and SPSS 25.0 were used for all calculations.

\section{Results}

\section{Study subjects}

Eighteen children with $\mathrm{PH}$ ( $<18$ years of age), 10 male, 8 female (median age $8.5 \mathrm{y}$, min.: $0.6 \mathrm{y}$, max.: $16.8 \mathrm{y}$ ), with a median weight of $21.3 \mathrm{~kg}$, min.: $5.0 \mathrm{~kg}$, max.: $106.5 \mathrm{~kg}$ and a median length of $124.3 \mathrm{~cm}$, min.: $55 \mathrm{~cm}$, max.: $192 \mathrm{~cm}$ at study start, were analyzed. Of those, 13 children were $<12$ y of age and two were infants $<1 \mathrm{y}$ of age (Table 1). All children were assigned a clinical $\mathrm{PH}$ group (subgroup definition in patients with CHD was performed according to the CHD defect prior to operation), based on the updated classification of pediatric $\mathrm{PH}(3,35)$. Eleven patients had $\mathrm{PH}$ associated with CHD. Those 11 children were divided in Group 1.4.4: CHD, i.e., children with simple operable left to right shunt $(n=3)$ and Group 5.4: children with complex CHD $(n=8)$. All of the CHD children have had a complete biventricular repair 


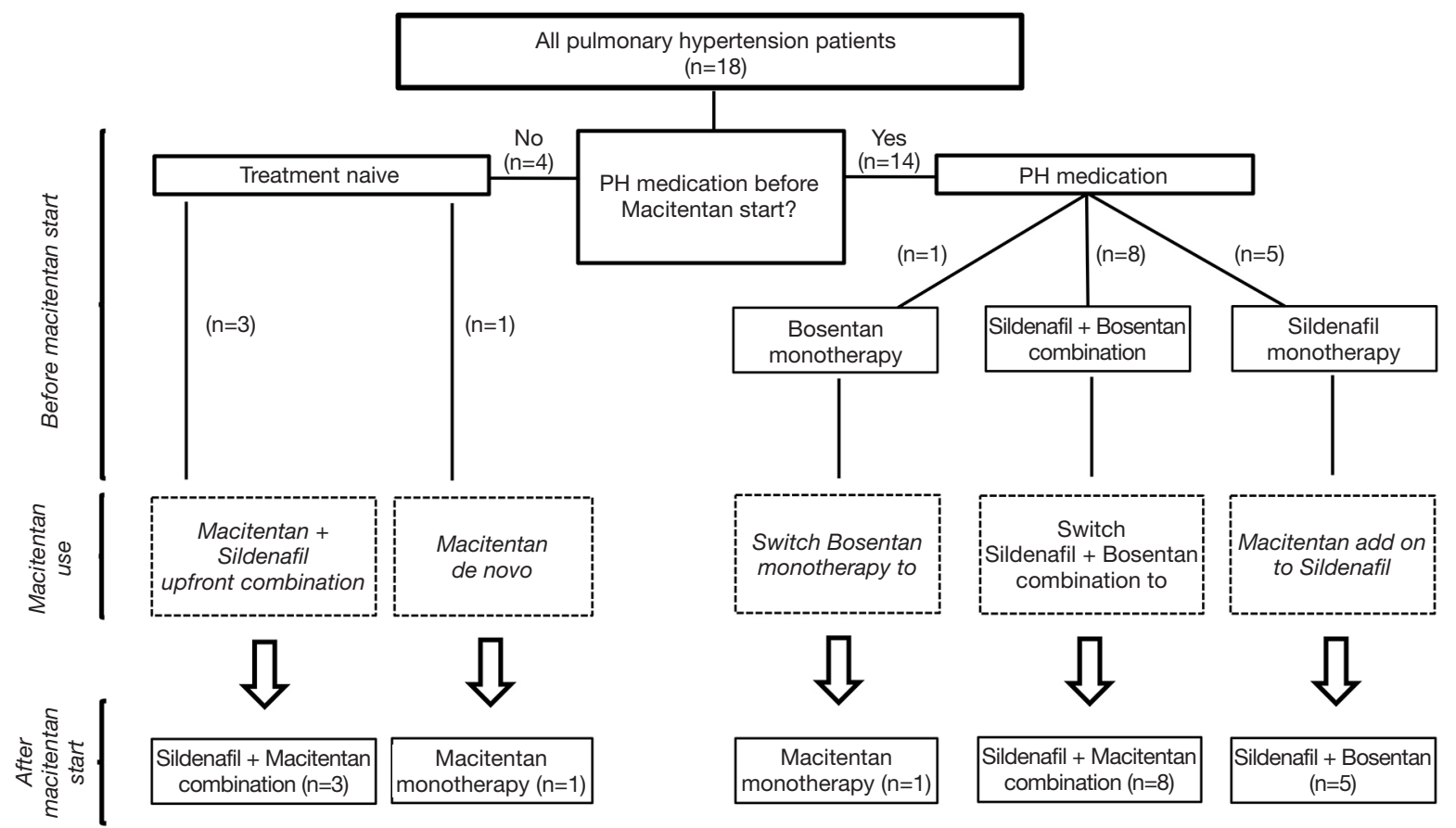

Figure 1 Treatment of pulmonary hypertension. PH, pulmonary hypertension; n, number.

of their CHD before macitentan was started (no patient with Fontan physiology). Five patients had $\mathrm{PH}$ associated with bronchopulmonary dysplasia (PH-BPD) (Group 3.5 PH), 1 girl had co-morbidity of an Abernethy malformation type $\mathrm{Ib}$ and portal hypertension (Group 1.4.3 $\mathrm{PH}$ ) and one patient had IPAH (Group 1.1 PH). Demographic data are outlined in Table 1.

\section{Treatment before macitentan start}

Four out of $18 \mathrm{PH}$ patients had no previous treatment (treatment naïve). Fourteen of 18 children were already on PH therapy: 8 children had a bosentan/sildenafil combination therapy, 1 child received bosentan monotherapy and 5 patients were on sildenafil monotherapy (Figure 1 and Table 1). The median treatment duration before the start of the macitentan treatment was 40 mo for bosentan $(n=9$, min.: 17 , max.: 79) and 34 mo for sildenafil (n=13, min.:1, max.: 79).

Monotherapy (with sildenafil or bosentan) was pursued in children belonging to the lower risk $\mathrm{PH}$ group. Initial combination therapy was used in children with an intermediate $\mathrm{PH}$ risk condition. No child had a contraindication for combination therapy. When patients were not stable or worsened under monotherapy (sildenafil or bosentan monotherapy) sequential add on therapy was used as recommended by the European Pediatric Pulmonary Vascular Disease Network (35). Children with previous $\mathrm{PH}$ medication had no change in medication 3 months prior to macitentan initiation.

\section{Macitentan treatment}

We analyzed only those pediatric patients who received oral macitentan for at least 4 weeks, one patient discontinued macitentan therapy already after two weeks due to subjective clinical worsening, headache and vertigo. Three patients who were treatment naïve received upfront combination therapy consisting of sildenafil and macitentan and were included in the adverse events assessment. The preliminary efficacy analysis of macitentan in $\mathrm{PH}$ was performed in 15 patients with a median follow-up of 6 months. Of the patients with previous $\mathrm{PH}$ therapy 5 received macitentan as an add-on therapy to sildenafil, while 8 children on combination therapy with bosentan plus sildenafil were switched to macitentan plus sildenafil. One child on bosentan monotherapy was switched to macitentan monotherapy (Figure 1, Table 1). In patients at intermediate $\mathrm{PH}$ risk, upfront combination therapy was initiated $(\mathrm{n}=3)$ (Figure 1). De novo monotherapy with macitentan was started in one patient with lower PH risk (this patient had 


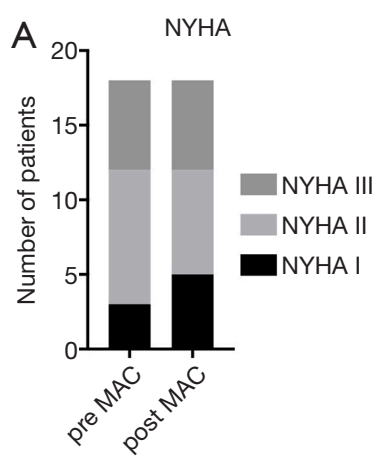

B

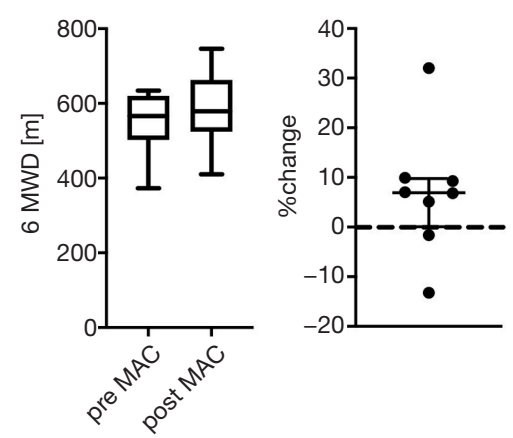

Figure 2 Clinical assessment. (A) Distribution of the New York Heart Association (NYHA) Functional Classification (FC) of all children ( $\mathrm{n}=18$ ) pre and post macitentan (MAC) start. (B) Six-minute walking distance (6MWD) in meters $(\mathrm{m})$ pre and post macitentan (MAC) start. Left: absolute values. The box and whisker plots (left) show the median, IQR and range. Right: percent change from baseline. The scatter plots (right) show the median with IQR.

no contraindication for combination therapy) (Figure 1). Children who were stable under mono or combination therapy (bosentan plus sildenafil) were switched to mono macitentan $(\mathrm{n}=1)$ or combination therapy (macitentan plus sildenafil, $\mathrm{n}=8$ ). Children who deteriorate under monotherapy with sildenafil received add on therapy with macitentan, $\mathrm{n}=5$ (Figure 1).

\section{Clinical assessment}

Before the start of macitentan, 3 children were in NYHA $\mathrm{FC} /$ modified ROSS score I (16.7\%), 9 patients in NYHA $\mathrm{FC} /$ modified ROSS score II $(50 \%)$ and 6 patients in NYHA FC/modified ROSS score III (33.3\%) (Figure 2A). No differences were seen between genders at the baseline clinical assessment. During the observation period on macitentan, NYHA FC/modified ROSS score remained unchanged in 16 patients (patients in NYHA FC/modified ROSS score I and III) and improved in 2 patients from NYHA FC/modified ROSS score II to I (Figure 2A).

\section{MWD}

The $6 \mathrm{MWD}$ tended to increase $(+7 \%)$ with macitentan in PH patients ( $\mathrm{n}=8$; baseline: median 567 meters, min.: 373, max.: 634 meters versus median 579, min.: 410, max.: 746 meters at follow-up, $\mathrm{P}>0.05$, Figure $2 B$ ).

\section{Echocardiography}

After initiation of macitentan, the estimated RVSP decreased $(-7 \%)$ from median 55 (min.: 30 , max.: 106) to median 45 (min.: 20, max.: 100) $\mathrm{mmHg}(\mathrm{P}<0.05)$. Longitudinal systolic RV function, as assessed by TAPSE, improved from median 1.4 (min.: 0.8 , max.: 2.8 ) to median 1.9 (min.: 0.8 , max.: 2.7$) \mathrm{cm}(\mathrm{P}<0.05,+20 \%)$. The PAAT did increase $(+4 \%)$ slightly from median 91 (min.: 50, max.: 120) to median 96 (min.: 66, max.: 120) ms, however, this change did not reach statistical significance $(\mathrm{P}>0.05)$. Results of echocardiography are expressed in Figure 3.

\section{Cardiac catheterization}

In 8 children, cardiac catherization was performed before and after the initiation of macitentan treatment. The PVRi improved (Figure 4A) from a median of 7.6 (min.:3.3, max.: 11.5 ) to 4.8 (min.: 2.5, max.: 10) Wood units $(\mathrm{WU}) \times \mathrm{m}^{2}$ body surface area $(\mathrm{BSA}) ; \mathrm{P}<0.05 ;-34 \%$. The $\mathrm{mPAP} / \mathrm{mSAP}$ ratio decreased $(\mathrm{P}<0.05 ;-27 \%)$ from a median of $62 \%$ (min.: $30 \%$, max.: $87 \%$ ) to $49 \%$ (min.: $30 \%$, max.: $69 \%$ ) (Figure $4 B$ ). The mPAP did not change significantly [baseline: $37.5 \mathrm{mmHg}$ (min.: 29, max.: 77), at follow-up: $38 \mathrm{mmHg}$ (min.: 17, max.: 46), $\mathrm{P}>0.05$; $-8 \%$, Figure $4 C]$ ] The CI did not change significantly [baseline median: $3.35 \mathrm{~L} / \mathrm{min} / \mathrm{m}^{2}$ (min.: 2.9 , max.: 6.1) versus follow-up median: $3.85 \mathrm{~L} / \mathrm{min} / \mathrm{m}^{2}$ (min.: 3.1 , max.: 8.3 ), $\mathrm{P}>0.05 ;-13 \%$ ].

\section{Laboratory findings}

During clinical follow-up, routine laboratory tests were performed at least twice after the start of the therapy in order to evaluate adverse drug effects. NT-proBNP values decreased significantly from $271.5 \mathrm{pg} / \mathrm{mL}$ (min.: 27, max.: 2,010) to 229 (min.: 23, max.: 814) pg/mL with macitentan therapy (Figure $5, \mathrm{P}<0.05 ;-41 \%$ ). In 

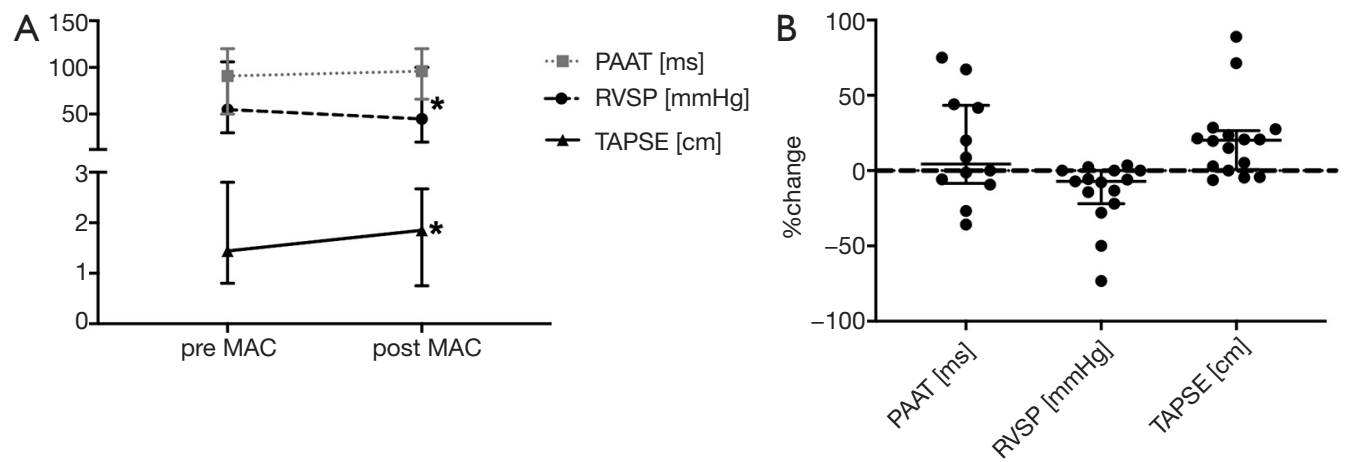

Figure 3 Echocardiography. (A) Trend of pulmonary acceleration time (PAAT), estimated right ventricular systolic pressure gradient (RVSP) and tricuspid annular plane systolic excursion (TAPSE) pre and post macitentan (MAC) start. The box and whisker plots (left) show the median, IQR and range. (B) PAAT, RVSP and TAPSE shown as percent change from baseline (before macitentan therapy). The scatter plots (right) show the median with IQR. *, significant change $\mathrm{P}<0.05$.
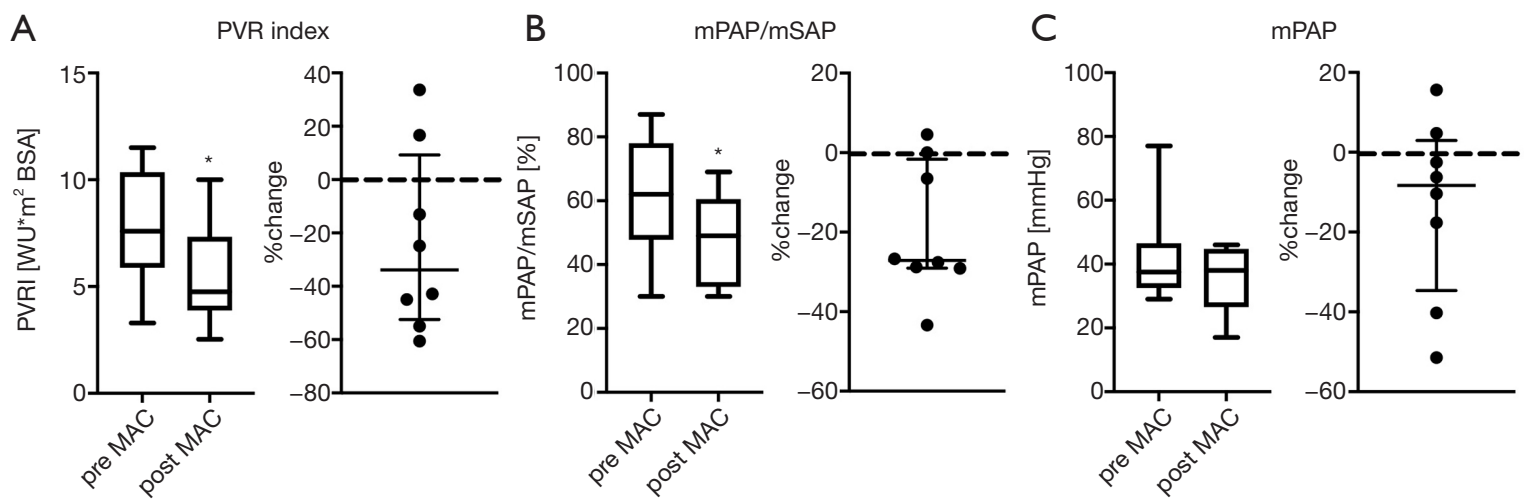

Figure 4 Invasive hemodynamics. (A) Left: the pulmonary vascular resistance index (PVRI) in Wood units (WU) $\times \mathrm{m}^{2}$ Body surface area (BSA) before (per) and after (post) macitentan (MAC) initiation. The box and whisker plots (left) show the median, IQR and range. Right: percent change from baseline (before macitentan therapy). The scatter plots (right) show the median with IQR. (B) Left: ratio of mean pulmonary artery pressure/mean systemic artery pressure (mPAP/mSAP ratio) in percent (\%) before (pre) and after (post) macitentan initiation. The box and whisker plots (left) show the median, IQR and range. Right: percent change from baseline (before macitentan therapy). The scatter plots (right) show the median with IQR. (C) Left: mean pulmonary artery pressure (mPAP) in millimeters of mercury (mmHg) before (pre) and after (post) macitentan initiation. The box and whisker plots (left) show the median, IQR and range. Right: percent change from baseline (before MAC therapy). The scatter plots (right) show the median with IQR. * significant change $\mathrm{P}<0.05$.

the SERAPHIN trial (19), the incidence of hepatoxicity and peripheral edema was similar in the macitentan and placebo group. Neither peripheral edema nor increases in aspartate aminotransferase, alanine aminotransferase, gamma-glutamyltransferase, or bilirubin were observed in our pediatric cohort. The most common and frequent laboratory abnormality with ERA including macitentan (19) is a decrease in hemoglobin. However, throughout our complete study period, no anemia was observed in our patients treated with macitentan.

\section{Discussion}

In 2013, the FDA approved macitentan (20), the new unselective ERA and pulmonary vasodilator and successor to bosentan. Macitentan exhibits enhanced receptor binding (15) and tissue penetration (16) as compared to bosentan. Macitentan improved mortality and morbidity in the placebo controlled SERAPHIN trial in a wide spectrum of PAH (19), including repaired CHD shunts. Macitentan received a IB recommendation for 


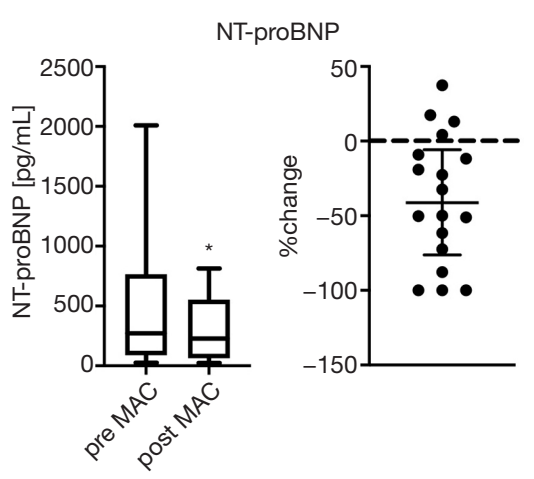

Figure 5 NT-proBNP. Left: absolute values of NT-proBNP. The box and whisker plots (left) show the median, IQR and range. Right: percent change from baseline (before macitentan therapy). The scatter plots (right) show the median with IQR. *, significant change $\mathrm{P}<0.05$. NT-proBNP, $\mathrm{N}$-terminal pro-brain natriuretic peptide.

monotherapy or sequential combination therapy and a IIA-C recommendation for initial drug combination of adult PAH patients in WHO-FC II and III in the most recent European ESC/ERS guidelines for PH (4). In the last two decades, $\mathrm{PH}$ prognosis has improved, coinciding with the introduction of new PAH-targeted therapies (36) and probably also due to more accurate diagnosis and early initiation of combination pharmacotherapy (1,36-39).

To the best of our knowledge, this is the first prospective study documenting effects of macitentan treatment in infants and young children $<12$ years of age. We performed echocardiography and obtained invasive hemodynamic data, laboratory results and clinical data before the initiation of macitentan and observed these parameters up to a median follow-up of 6 months. In the present study, treatment with macitentan was pursued at a once-daily dose of $5 \mathrm{mg}$ (<10 kg body weight) and $10 \mathrm{mg}$ (>10 kg body weight). Such treatment over a long observational time of up to 30 months resulted in significant improvements in invasive hemodynamic variables (PVRi, $\mathrm{mPAP} / \mathrm{mSAP}$ ratio) and NT-proBNP levels. Similar observations were reported in a hemodynamic sub-study of the SERAPHIN trial (40).

In our study, pediatric $\mathrm{PH}$ patients treated with macitentan were found to have improved RVSP and longitudinal systolic RV function (TAPSE) versus baseline, as assessed by echocardiography. In 43 adults with PAHCHD, a switch from bosentan to macitentan was associated with improvements in NT-pro-BNP, TAPSE and WHO
FC after 6 months follow-up, even after several years of bosentan (23\% were on bosentan/sildenafil combination at study start) (41). Safdar et al. reported that a rapid switch from bosentan to macitentan (24 adult PAH patients, PAHCHD, $\mathrm{n}=5$ ) maintained WHO FC and NT-pro-BNP levels (42). However, Tynan et al. (43) showed that in a group of adult PAH patients with poorly controlled disease on existing bosentan or ambrisentan $(\mathrm{n}=37)$, switching to a different ERA (macitentan) or adding macitentan to preexisting sildenafil resulted in a significant improvement in WHO FC. Our study was very likely underpowered to detect significant improvements in NYHA FC/modified ROSS score. We observed no significant improvement of 6MWD, as Safdar et al. (42) and Blok et al. (41) previously reported. However, in another study, macitentan improved the $6 \mathrm{MWD}$, but not the NT-pro-BNP levels, systolic pulmonary artery pressure estimated by echocardiography, or WHO FC (25). In contrast, Tynan et al. (43) reported significant improvements in $6 \mathrm{MWD}$ in the macitentan addon group, but not in the bosentan-to-macitentan-switch group.

In our study, one patient discontinued macitentan therapy already after two weeks due to subjective clinical worsening, headache and vertigo. In all other patients, macitentan treatment was well tolerated and during our observational time no hepatotoxicity, liver failure or drop in hemoglobin was seen in our pediatric cohort. Several children with $\mathrm{PH}$ in our study were changed from bosentan to macitentan because of the reported less side effects of this drug and the administration frequency (once per day). Usually bosentan is administered twice a day, going along with difficulties in implementation of the application in daily routine, which is a major problem for parents and patients. The beneficial profile of macitentan with once per day dosing, probably increases patient compliance and makes macitentan an appealing ERA to use in PAH.

\section{Limitations of the study}

Our cohort is small, heterogenic in age, etiologies and therapeutic strategies, i.e., pharmacotherapy at baseline, and includes patients on diverse $\mathrm{PH}$ medications. However, this study reflects real life situations faced by pediatric cardiologists in daily practice. Invasive measurements could not be performed in all patients due to low weight and size of the infants, small vessels (risk of vessel injury with consecutive vessel perforation and obstruction) and/or lack 
of caregiver consent.

\section{Conclusions}

This is the first prospective, exploratory study on $\mathrm{PH}$ targeted pharmacotherapy with macitentan in infants and young children $<12$ years of age. Macitentan was associated with improvements in invasive hemodynamics (mPAP/ mSAP, PVRi), echocardiographic variables of RV systolic function (TAPSE) and serum NT-pro-BNP concentrations. These findings in a small number of children suggest the potential for clinical safety and efficacy of macitentan in children with $\mathrm{PH}$. Future controlled and randomized studies on macitentan with more pediatric participants, more stringent $\mathrm{PH}$ subgroup definitions, and predefined and extended follow-up protocols are needed and currently ongoing [the Phase III study TOMORROW study aims to delineate pharmacokinetics, best dosing and the long-term benefits of Macitentan $\left(\right.$ Opsumit $\left.^{\circledR}\right)$ in children with PAH (NCT02932410)].

\section{Acknowledgments}

Funding: GH receives funding from the German Research Foundation (DFG; HA4348/2-2, HA4348/6-2 KFO311).

\section{Footnote}

Provenance and Peer Review: This article was commissioned by the Guest Editors (Martin Koestenberger, Harm-Jan Bogaard and Georg Hansmann) for the series "Right Ventricular Dysfunction" published in Cardiovascular Diagnosis and Therapy. The article was sent for external peer review organized by the Editor-in-Chief and the editorial office.

Conflicts of Interest: All authors have completed the ICMJE uniform disclosure form (available at http:// dx.doi.org/10.21037/cdt.2020.04.01). The series "Right Ventricular Dysfunction" was commissioned by the editorial office without any funding or sponsorship. AG reports grants from JNJ during the conduct of the study. Actelion/ Johnson\&Johnson had no influence on this publication, has not seen the data or the manuscript and had no role in the production of this manuscript. The authors are solely responsible for the design and conduction of this study, all study analyses, the drafting and editing of the paper and its final contents. The authors have no other conflicts of interest to declare.
Ethical Statement: The authors are accountable for all aspects of the work in ensuring that questions related to the accuracy or integrity of any part of the work are appropriately investigated and resolved. The study was conducted in accordance with the Declaration of Helsinki (as revised in 2013). The study was approved by the ethics committee of the Medical University of Graz (No. 30-477 ex 17/18). Written informed consent was obtained from the legal caregivers of the patients and the patients for publication of this study and any accompanying images.

Open Access Statement: This is an Open Access article distributed in accordance with the Creative Commons Attribution-NonCommercial-NoDerivs 4.0 International License (CC BY-NC-ND 4.0), which permits the noncommercial replication and distribution of the article with the strict proviso that no changes or edits are made and the original work is properly cited (including links to both the formal publication through the relevant DOI and the license). See: https://creativecommons.org/licenses/by-nc-nd/4.0/.

\section{References}

1. Hansmann G. Pulmonary hypertension in infants, children, and young adults. J Am Coll Cardiol 2017;69:2551-69.

2. Rosenzweig EB, Abman SH, Adatia I, et al. Paediatric pulmonary arterial hypertension: updates on definition, classification, diagnostics and management. Eur Respir J 2019;53:1801916.

3. Simonneau G, Montani D, Celermajer DS, et al. Haemodynamic definitions and updated clinical classification of pulmonary hypertension. Eur Respir J 2019;53:1801913.

4. Galiè N, Humbert M, Vachiery JL, et al. 2015 ESC/ERS Guidelines for the diagnosis and treatment of pulmonary hypertension: The Joint Task Force for the Diagnosis and Treatment of Pulmonary Hypertension of the European Society of Cardiology (ESC) and the European Respiratory Society (ERS): Endorsed by: Association for European Paediatric and Congenital Cardiology (AEPC), International Society for Heart and Lung Transplantation (ISHLT). Eur Heart J 2016;37:67-119.

5. van Loon RL, Roofthooft MT, Hillege HL, et al. Pediatric pulmonary hypertension in the Netherlands: epidemiology and characterization during the period 1991 to 2005 . Circulation 2011;124:1755-64.

6. Fraisse A, Jais X, Schleich JM, et al. Characteristics and prospective 2-year follow-up of children with pulmonary 
arterial hypertension in France. Arch Cardiovasc Dis 2010;103:66-74.

7. Moledina S, Hislop AA, Foster H, et al. Childhood idiopathic pulmonary arterial hypertension: a national cohort study. Heart 2010;96:1401-6.

8. D'Alonzo GE, Barst RJ, Ayres SM, et al. Survival in patients with primary pulmonary hypertension. Results from a national prospective registry. Ann Intern Med 1991;115:343-9.

9. Barst RJ, McGoon MD, Elliott CG, et al. Survival in childhood pulmonary arterial hypertension: insights from the registry to evaluate early and long-term pulmonary arterial hypertension disease management. Circulation 2012;125:113-22.

10. van Loon RL, Roofthooft MT, Delhaas T, et al. Outcome of pediatric patients with pulmonary arterial hypertension in the era of new medical therapies. Am J Cardiol 2010;106:117-24.

11. Galiè N, Rubin L, Hoeper M, et al. Treatment of patients with mildly symptomatic pulmonary arterial hypertension with bosentan (EARLY study): a double-blind, randomised controlled trial. Lancet 2008;371:2093-100.

12. Dingemanse J, Sidharta PN, Maddrey WC, et al. Efficacy, safety and clinical pharmacology of macitentan in comparison to other endothelin receptor antagonists in the treatment of pulmonary arterial hypertension. Expert Opin Drug Saf 2014;13:391-405.

13. Channick R, Badesch DB, Tapson VF, et al. Effects of the dual endothelin receptor antagonist bosentan in patients with pulmonary hypertension: a placebo-controlled study. J Heart Lung Transplant 2001;20:262-3.

14. Rubin LJ, Roux S. Bosentan: a dual endothelin receptor antagonist. Expert Opin Investig Drugs 2002;11:991-1002.

15. Gatfield J, Mueller Grandjean C, Sasse T, et al. Slow receptor dissociation kinetics differentiate macitentan from other endothelin receptor antagonists in pulmonary arterial smooth muscle cells. PLoS One 2012;7:e47662.

16. Iglarz M, Binkert C, Morrison K, et al. Pharmacology of macitentan, an orally active tissue-targeting dual endothelin receptor antagonist. J Pharmacol Exp Ther 2008;327:736-45.

17. Sidharta PN, van Giersbergen PL, Halabi A, et al. Macitentan: entry-into-humans study with a new endothelin receptor antagonist. Eur J Clin Pharmacol 2011;67:977-84.

18. Sidharta PN, van Giersbergen PL, Dingemanse J. Safety, tolerability, pharmacokinetics, and pharmacodynamics of macitentan, an endothelin receptor antagonist, in an ascending multiple-dose study in healthy subjects. J Clin Pharmacol 2013;53:1131-8.

19. Pulido T, Adzerikho I, Channick RN, et al. Macitentan and morbidity and mortality in pulmonary arterial hypertension. N Engl J Med 2013;369:809-18.

20. Patel T, McKeage K. Macitentan: first global approval. Drugs 2014;74:127-33.

21. Khadka A, Singh Brashier DB, Tejus A, et al. Macitentan: An important addition to the treatment of pulmonary arterial hypertension. J Pharmacol Pharmacother 2015;6:53-7.

22. Paul GA, Gibbs JS, Boobis AR, et al. Bosentan decreases the plasma concentration of sildenafil when coprescribed in pulmonary hypertension. Br J Clin Pharmacol 2005;60:107-12.

23. Grünig E, Haefeli WE. Plasma Drug Concentrations in Children Receiving Pulmonary Arterial Hypertension Combination Treatment. Respiration 2019;97:92.

24. Enderby CY, Burger C. Medical treatment update on pulmonary arterial hypertension. Ther Adv Chronic Dis 2015;6:264-72.

25. Aypar E, Alehan D, Karagoz T, et al. Clinical efficacy and safety of switch from bosentan to macitentan in children and young adults with pulmonary arterial hypertension. Cardiol Young 2018;28:542-7.

26. Gatzoulis MA, Landzberg M, Beghetti M, et al. Evaluation of Macitentan in Patients With Eisenmenger Syndrome. Circulation 2019;139:51-63.

27. Ross RD. The Ross classification for heart failure in children after 25 years: a review and an age-stratified revision. Pediatr Cardiol 2012;33:1295-300.

28. Masarone D, Valente F, Rubino M, et al. Pediatric Heart Failure: A Practical Guide to Diagnosis and Management. Pediatr Neonatol 2017;58:303-12.

29. ATS Committee on Proficiency Standards for Clinical Pulmonary Function Laboratories. ATS statement: guidelines for the six-minute walk test. Am J Respir Crit Care Med 2002;166:111-7.

30. Lopez L, Colan SD, Frommelt PC, et al.

Recommendations for quantification methods during the performance of a pediatric echocardiogram: a report from the Pediatric Measurements Writing Group of the American Society of Echocardiography Pediatric and Congenital Heart Disease Council. J Am Soc Echocardiogr 2010;23:465-95; quiz 576-7.

31. Koestenberger M, Ravekes W, Everett AD, et al. Right ventricular function in infants, children and adolescents: reference values of the tricuspid annular plane systolic 
excursion (TAPSE) in 640 healthy patients and calculation of z score values. J Am Soc Echocardiogr 2009;22:715-9.

32. Koestenberger M, Grangl G, Avian A, et al. Normal Reference Values and z Scores of the Pulmonary Artery Acceleration Time in Children and Its Importance for the Assessment of Pulmonary Hypertension. Circ Cardiovasc Imaging 2017. doi: 10.1161/CIRCIMAGING.116.005336.

33. McQuillan BM, Picard MH, Leavitt M, et al. Clinical correlates and reference intervals for pulmonary artery systolic pressure among echocardiographically normal subjects. Circulation 2001;104:2797-802.

34. Bossone E, D'Andrea A, D'Alto M, et al. Echocardiography in pulmonary arterial hypertension: from diagnosis to prognosis. J Am Soc Echocardiogr 2013;26:1-14.

35. Hansmann G, Koestenberger M, Alastalo TP, et al. 2019 updated consensus statement on the diagnosis and treatment of pediatric pulmonary hypertension: The European Pediatric Pulmonary Vascular Disease Network (EPPVDN), endorsed by AEPC, ESPR and ISHLT. J Heart Lung Transplant 2019;38:879-901.

36. Beghetti M, Gorenflo M, Ivy DD, et al. Treatment of pediatric pulmonary arterial hypertension: A focus on the NO-sGC-cGMP pathway. Pediatr Pulmonol 2019;54:1516-26.

37. Galiè N, Channick RN, Frantz RP, et al. Risk stratification and medical therapy of pulmonary arterial hypertension. Eur Respir J 2019. doi: 10.1183/13993003.01889-2018.

38. Lammers AE, Apitz C, Zartner P, et al. Diagnostics, monitoring and outpatient care in children with

Cite this article as: Schweintzger S, Koestenberger M, Schlagenhauf A, Grangl G, Burmas A, Kurath-Koller S, Pocivalnik M, Sallmon H, Baumgartner D, Hansmann G, Gamillscheg A. Safety and efficacy of the endothelin receptor antagonist macitentan in pediatric pulmonary hypertension. Cardiovasc Diagn Ther 2020;10(5):1675-1685. doi: 10.21037/ cdt.2020.04.01 suspected pulmonary hypertension/paediatric pulmonary hypertensive vascular disease. Expert consensus statement on the diagnosis and treatment of paediatric pulmonary hypertension. The European Paediatric Pulmonary Vascular Disease Network, endorsed by ISHLT and DGPK. Heart 2016;102 Suppl 2:ii1-13.

39. Hansmann G, Apitz C. Treatment of children with pulmonary hypertension. Expert consensus statement on the diagnosis and treatment of paediatric pulmonary hypertension. The European Paediatric Pulmonary Vascular Disease Network, endorsed by ISHLT and DGPK. Heart 2016;102 Suppl 2:ii67-85.

40. Galiè N, Jansa P, Pulido T, et al. SERAPHIN haemodynamic substudy: the effect of the dual endothelin receptor antagonist macitentan on haemodynamic parameters and NT-proBNP levels and their association with disease progression in patients with pulmonary arterial hypertension. Eur Heart J 2017;38:1147-55.

41. Blok IM, van Riel A, van Dijk APJ, et al. From bosentan to macitentan for pulmonary arterial hypertension and adult congenital heart disease: Further improvement? Int J Cardiol 2017;227:51-2.

42. Safdar Z, Thakur A, Frost A. Tolerability of Switch to Macitentan from Bosentan in Pulmonary Arterial Hypertension. South Med J 2017;110:223-8.

43. Tynan T, Hird K, Hannon T, et al. Pulmonary arterial hypertension outcomes upon endothelin-1 receptor antagonist switch to macitentan. J Int Med Res. 2019;47:2177-86. 